\title{
Central Nervous System Embryonal Tumor, Not Otherwise Specified with Leptomeningeal Spread
}

National Cancer Institute

\section{Source}

National Cancer Institute. Central Nervous System Embryonal Tumor, Not Otherwise Specified with Leptomeningeal Spread. NCI Thesaurus. Code C5400.

A central nervous system embryonal tumor, not otherwise specified which has spread from its original site to the leptomeninges surrounding the brain and spinal cord. 